\title{
Converse Sturm-Hurwitz-Kellogg theorem and related results
}

\author{
Serge Tabachnikov
}

To V. I. Arnold for his 70th birthday

\begin{abstract}
We prove that if $V^{n}$ is a Chebyshev system on the circle and $f$ is a continuous real-valued function with at least $n+1$ sign changes then there exists an orientation preserving diffeomorphism of $S^{1}$ that takes $f$ to a function $L^{2}$-orthogonal to $V$. We also prove that if $f$ is a function on the real projective line with at least four sign changes then there exists an orientation preserving diffeomorphism of $\mathbb{R P}^{1}$ that takes $f$ to the Schwarzian derivative of a function on $\mathbb{R P}^{1}$. We show that the space of piecewise constant functions on an interval with values \pm 1 and at most $n+1$ intervals of constant sign is homeomorphic to $n$-dimensional sphere.
\end{abstract}

Mathematics Subject Classification (2000). 53A04, 42A70.

Keywords. 4-vertex theorem, Sturm-Hurwitz-Kellogg theorem, Ghys theorem, Chebyshev system, Schwarzian derivative.

\section{Introduction and formulation of results}

The classical four vertex theorem asserts that the curvature of a plane oval (strictly convex smooth closed curve) has at least four extrema. Discovered about 100 years ago by S. Mukhopadhyaya, this theorem and its numerous generalizations and refinements continue to attract attention up to this day; see [7] for a sampler.

One such result is the converse four vertex theorem proved by Gluck for strictly convex, and by Dahlberg for general curves $[3,1]$ : a periodic function having at least two local minima and two local maxima is the curvature function of a simple closed plane curve. See [2] for a very well written survey.

The radius of curvature $\rho(\alpha)$ of an oval, considered as a function of the direction of the tangent line to the curve, is $L^{2}$-orthogonal to the first harmonics:

$$
\int_{0}^{2 \pi} \rho(\alpha) \cos \alpha d \alpha=\int_{0}^{2 \pi} \rho(\alpha) \sin \alpha d \alpha=0
$$


Such a function must have at least four critical points. The converse four vertex theorem can be restated as follows: if a function $\rho(\alpha)$ has at least two local minima and two local maxima then there is a diffeomorphism $\varphi$ of the circle such that the function $\rho(\varphi(\alpha))$ is $L^{2}$-orthogonal to the first harmonics.

Our first result is the following generalization.

A Chebyshev system is an $n$-dimensional ${ }^{1}$ space $V$ of functions on the circle $S^{1}=\mathbb{R} / 2 \pi \mathbb{Z}$ such that every non-zero function from $V$ has at most $n-1$ zeros (counted with multiplicities). According to the Sturm-Hurwitz-Kellogg theorem, if a smooth function ${ }^{2}$ on $S^{1}$ is $L^{2}$-orthogonal to a Chebyshev system $V^{n}$ then this function has at least $n+1$ sign changes; see, e.g., [7]. In particular, a function orthogonal to $\{1, \cos \alpha, \sin \alpha\}$ has at least four zeros; applied to the derivative of the radius of curvature of an oval, this implies the four vertex theorem.

We prove the converse Sturm-Hurwitz-Kellogg theorem.

Theorem 1. Let $V^{n}$ be a Chebyshev system on $S^{1}$. If $f$ is a continuous function on $S^{1}$ with at least $n+1$ sign changes then there exists an orientation preserving diffeomorphism $\varphi: S^{1} \rightarrow S^{1}$ such that $\varphi^{*}(f):=f \circ \varphi$ is $L^{2}$-orthogonal to $V$.

Our strategy of the proof is that of Gluck $[3,2]$ which we illustrate by the following simplest case of the above theorem.

Example 1.1. Let $f$ be a continuous function on $S^{1}$ that has both positive and negative values. One claims that there exists an orientation preserving diffeomorphism $\varphi: S^{1} \rightarrow S^{1}$ such that $f \circ \varphi$ has zero average value:

$$
\int_{0}^{2 \pi} f(\varphi(x)) d x=0 .
$$

Of course, this is obvious, but we shall describe an argument that exemplifies the method of proof of Theorem 1 and other results of this paper.

Step 1 . Let $h$ be the step function that takes value 1 on $[0, \pi)$ and -1 on $[\pi, 2 \pi)$. This step function has zero average value.

Step 2. Since $f$ changes sign, there is a number $c \neq 0$ such that $f$ assumes both values $\pm c$. Scaling $f$, assume that $c=1$ and that $f\left(x_{1}\right)=1, f\left(x_{2}\right)=-1$. For every $\varepsilon>0$, there exists a diffeomorphism $\varphi \in \operatorname{Diff}_{+}\left(S^{1}\right)$ which stretches neighborhoods of the points $x_{1}$ and $x_{2}$ so that $\varphi^{*}(f)$ is $\varepsilon$-close in measure to $h$.

Step 3. For a sufficiently small real $\alpha$, consider an orientation preserving diffeomorphism $\psi_{\alpha} \in \operatorname{Diff}_{+}\left(S^{1}\right)$ that fixes 0 and stretches the interval $[0, \pi]$ to $[0, \pi+\alpha]$. We assume that the dependence of $\psi_{\alpha}$ on $\alpha$ is smooth. The correspondence $\alpha \mapsto \psi_{\alpha}$ is a map of an interval $I$ to the group $\operatorname{Diff}_{+}\left(S^{1}\right)$. Consider the function

$$
F(\alpha)=\int_{0}^{2 \pi} \psi_{\alpha}^{*}(h)(x) d x .
$$

\footnotetext{
${ }^{1}$ Where $n$ is odd. One can define a Chebyshev system on an interval as well, and then there is no restriction on the parity of its dimension.

${ }^{2}$ Smoothness is not needed; one can work with finitely differentiable or continuous functions.
} 
One has $F(0)=0$ and $F^{\prime}(0) \neq 0$. In particular, making the interval $I$ smaller, if needed, $F$ has opposite signs at the end points of $I$.

Step 4. Finally, replace $h$ in the definition of $F$ by the function $\varphi^{*}(f)$ from Step 2. If $\varepsilon$ is small enough, the resulting function $\bar{F}: I \rightarrow \mathbb{R}$ still has opposite signs at the end points of $I$, hence there exists $\bar{\alpha}$ such that $\bar{F}(\bar{\alpha})=0$. Thus the function $\psi_{\bar{\alpha}}^{*}\left(\varphi^{*}(f)\right)$ has zero average.

Remark 1.2. An object invariantly related to a function is its differential $d f=$ $f^{\prime}(x) d x$ (rather than the derivative). If $\lambda$ is a differential 1-form on $S^{1}$ and

$$
\int_{0}^{2 \pi} \lambda=0
$$

then $\lambda$ has sign changes, but the converse does not hold since

$$
\int_{0}^{2 \pi} \varphi^{*}(\lambda)=\int_{0}^{2 \pi} \lambda
$$

for every $\varphi \in \operatorname{Diff}_{+}\left(S^{1}\right)$. This explains why we deal with a function, rather than a differential 1-form.

Another, rather recent, four-vertex-type theorem is due to E. Ghys: the Schwarzian derivative of a diffeomorphism of the real projective line has at least four zeros. Choose an affine coordinate $x$ on $\mathbb{R P}^{1}$ and let $f$ be a diffeomorphism. Then the Schwarzian derivative $S(f)$ is given by the formula

$$
S(f)=\frac{f^{\prime \prime \prime}}{f^{\prime}}-\frac{3}{2}\left(\frac{f^{\prime \prime}}{f^{\prime}}\right)^{2} ;
$$

it measures the failure of $f$ to preserve the projective structure; see [7].

We prove a converse theorem.

Theorem 2. If $f$ is a smooth function on $\mathbb{R}^{1}$ with at least four sign changes then there exists an orientation preserving diffeomorphism $\varphi$ of the projective line and $g$ such that $\varphi^{*}(f)=S(g)$.

Remark 1.3. The invariant meaning of the Schwarzian is not a function but rather a quadratic differential (see, e.g., [7] for a detailed discussion):

$$
S(f)=\left(\frac{f^{\prime \prime \prime}}{f^{\prime}}-\frac{3}{2}\left(\frac{f^{\prime \prime}}{f^{\prime}}\right)^{2}\right) d x^{2} .
$$

Similarly to Remark 1.2 , the property of a quadratic differential on $\mathbb{R P}^{1}$ to be the Schwarzian derivative of a diffeomorphism is invariant under the action of the $\operatorname{group} \operatorname{Diff}\left(\mathbb{R P}^{1}\right)$.

\section{Proof of the converse Sturm-Hurwitz-Kellogg theorem}

The proof consists of the same four steps as in Example 1.1. 
Step 1 .

Lemma 2.1. There exists a piecewise constant function on $S^{1}$ with values \pm 1 and exactly $n+1$ intervals of constant sign which is $L^{2}$-orthogonal to $V$.

Proof (suggested by D. Khavinson). Extend $V^{n}$ to a larger Chebyshev system $W^{n+2}$ and pick $f \in W-V$. Consider $g$, the best $L^{1}$ approximation of $f$ by a function in $V$. The function $g$ exists since $V$ is finite-dimensional.

Since $W$ is a Chebyshev system, $f-g$ has at most $n+1$ intervals of constant sign (obviously, $f-g \neq 0$ ). Let $I_{k}$ be these intervals, and let $h$ be the function that has alternating values \pm 1 on the intervals $I_{k}$. Since $g$ is the best approximation of $f$, one has the Lagrange multipliers condition:

$$
\left.\frac{d}{d \varepsilon}\right|_{\varepsilon=0}\left(\int_{0}^{2 \pi}|(f-g)(x)+\varepsilon v(x)| d x\right)=0
$$

for every $v \in V$. It follows from (1) that

$$
0=\sum_{k}(-1)^{k} \int_{I_{k}} v(x) d x=\int_{0}^{2 \pi} h(x) v(x) d x,
$$

that is, $h$ is orthogonal to $V$.

By the Sturm-Hurwitz-Kellogg theorem, $h$ has at least $n+1$ sign changes (proof, for completeness: if not, one can find a function from $V$ with the same intervals of constant sign as $h$; such a function cannot be orthogonal to $h$ ).

Step 2. Since $f$ changes sign at least $n+1$ times, there is a non-zero constant $c$ such that $f$ takes the alternating values $\pm c$ at points, say, $x_{0}, \ldots, x_{n}$. Multiplying $f$ by a constant, assume that $c=1$.

Let $h$ be the function from Lemma 2.1. For every $\varepsilon>0$, there exists a diffeomorphism $\varphi \in \operatorname{Diff}_{+}\left(S^{1}\right)$ which stretches neighborhoods of the points $x_{0}, \ldots, x_{n}$ so that the function $\varphi^{*}(f)$ is $\varepsilon$-close in measure to $h$.

Step 3. Consider the function $h$ and let $\left[0, x_{1}\right],\left[x_{1}, x_{2}\right], \ldots,\left[x_{n}, 2 \pi\right]$ be its intervals of constant sign. For $\alpha=\left(\alpha_{1}, \ldots, \alpha_{n}\right)$, consider an orientation preserving diffeomorphism $\psi_{\alpha} \in \operatorname{Diff}_{+}\left(S^{1}\right)$ that stretches the intervals $\left[x_{i}, x_{i+1}\right]$ so that point $x_{i}$ goes to $x_{i}+\alpha_{i}$ and which fixes 0 . We assume that each $\left|\alpha_{i}\right|$ is sufficiently small and that the dependence of $\psi_{\alpha}$ on $\alpha$ is smooth. The correspondence $\alpha \mapsto \psi_{\alpha}$ is a map of an $n$-dimensional disc $D^{n}$ to $\operatorname{Diff}_{+}\left(S^{1}\right)$.

The formula $F(\alpha)(g)=\left\langle\psi_{\alpha}^{*}(h), g\right\rangle$ defines a smooth map $D \rightarrow V^{*}$ that takes the origin to the origin (the scalar product is understood in the $L^{2}$ sense).

Lemma 2.2. The differential $d F$ is non-degenerate at the origin.

Proof. Let $g_{1}, \ldots, g_{n}$ be a basis of $V$. We want to prove that the matrix

$$
c_{i j}=\left.\frac{\partial F(\alpha)\left(g_{i}\right)}{\partial \alpha_{j}}\right|_{\alpha=0}, \quad i, j=1, \ldots, n,
$$


is non-singular. One has

$$
F(\alpha)(g)=\sum_{k=0}^{n}(-1)^{k} \int_{x_{k}+\alpha_{k}}^{x_{k+1}+\alpha_{k+1}} g(x) d x
$$

where we assume that $x_{0}=0, x_{n+1}=2 \pi, \alpha_{0}=\alpha_{n+1}=0$. It follows that $c_{i j}=2(-1)^{j+1} g_{i}\left(x_{j}\right)$, and it suffices to show that the matrix $g_{i}\left(x_{j}\right)$ is non-singular. This is indeed a fundamental property of Chebyshev systems, see [6] (proof, for completeness: if $c=\left(c_{1}, \ldots, c_{n}\right)$ is a non-zero vector such that $\sum c_{i} g_{i}\left(x_{j}\right)=0$ for each $j$ then the function $\sum c_{i} g_{i}$ has $n$ zeros, which contradicts the definition of Chebyshev systems).

Step 4. It follows from Lemma 2.2 that there exists $\delta>0$ such that the map $F$, restricted to the cube $D^{n}$ given by the conditions $\left|\alpha_{i}\right|<\delta, i=1, \ldots, n$, has degree one, and the hypersurface $F(\partial D)$ has rotation number one with respect to the origin in $V^{*}$.

Now replace $h$ in the definition of the map $F$ by the function $\varphi^{*}(f)$ from Step 2, and denote the new map by $\bar{F}: D^{n} \rightarrow V^{*}$. We shall be done if we show that there exists $\alpha$ such that $\bar{F}(\alpha)=0$. Indeed, if $\varepsilon$ is small enough then $\bar{F}(\partial D)$ still has rotation number one with respect to the origin in $V^{*}$, and therefore $\bar{F}(D)$ contains the origin.

\section{Digression: the space of step functions with values \pm 1 on an interval}

An extension of Lemma 2.1 to the case when $V$ is not assumed to be a Chebyshev system is the following Hobby-Rice theorem [4] (see also [10, 12]).

Theorem 3. Let $V$ be an $n$-dimensional subspace in $L^{1}([0,1])$. Then there exists a piecewise constant function on I with values \pm 1 and at most $n+1$ intervals of constant sign which is $L^{2}$-orthogonal to $V$.

Proof $([4,10])$. Let $x=\left(x_{0}, x_{1}, \ldots, x_{n}\right), \sum_{i} x_{i}^{2}=1$, be a point of the sphere $S^{n}$. Assign to $x$ the partition of $[0,1]$ into intervals of consecutive lengths $x_{0}^{2}, \ldots, x_{n}^{2}$ and the piecewise constant function $h_{x}$ with value equal to $\operatorname{sign} x_{i}$ on the respective interval. We obtain a map $F: S_{n} \rightarrow V^{*}$ given by the formula

$$
\langle F(x), g\rangle=\int_{0}^{1} h_{x}(t) g(t) d t .
$$

This map is odd: $F(-x)=-F(x)$, and it follows from the Borsuk-Ulam theorem (see, e.g., [5]) that $F(x)=0$ for some $x \in S_{n}$. Thus $h_{x}$ is orthogonal to $V$.

From the point of view of topology, it is interesting to consider the space $S_{n} \subset L^{1}([0,1])$ of piecewise constant functions on $[0,1]$ with values \pm 1 and at most $n+1$ intervals of constant sign. We complement the proof of Theorem 3 with the following result. 
Theorem 4. $S_{n}$ is homeomorphic to $n$-dimensional sphere.

Proof. We give $S_{n}$ the structure of a finite cell complex with two cells in every dimension $0,1, \ldots, n$ and prove, by induction on $n$, that $S_{n}$ is homeomorphic to $S^{n}$. For $n=0$, the set $S_{0}$ consists of two constant functions with values +1 or -1 and is homeomorphic to $S^{0}$.

Let $\Delta^{n}=\left\{x=\left(x_{0}, \ldots, x_{n}\right) \mid x_{i} \geq 0, \sum x_{i}=1\right\}$ be the standard simplex. Consider the subset $C \subset S_{n}$ consisting of functions with exactly $n+1$ intervals of constant sign. The lengths of these intervals are positive numbers $x_{0}, x_{1}, \ldots, x_{n}$ satisfying $\sum x_{i}=1$, and a function from $C$ is determined by $x=\left(x_{0}, \ldots, x_{n}\right)$ and the sign \pm that the function has on the first interval. Thus we obtain two embeddings $\psi_{ \pm}^{n}$ : Int $\Delta^{n} \rightarrow C$, and $C$ is the disjoint union of the images of $\psi_{+}^{n}$ and $\psi_{-}^{n}$.

The maps $\psi_{ \pm}^{n}$ extend continuously to the boundary $\partial \Delta^{n}$ : when some $x_{i} \mathrm{~s}$ shrink to zero, the respective intervals of constant sign of a function disappear, and if the function has the same signs in neighboring segments, they merge together. For example, let $n=2$. Then $\psi_{+}^{2}\left(0, x_{1}, x_{2}\right)$ has two intervals of constant sign and equals $\psi_{-}^{1}\left(x_{1}, x_{2}\right)$, whereas $\psi_{+}^{2}\left(x_{0}, 0, x_{2}\right)$ is the constant function with value +1 , i.e., equals $\psi_{+}^{0}(1)$.

We have $S_{n}-C=S_{n-1}$, and the latter is homeomorphic to $S^{n-1}$ by the induction assumption. Each map $\psi_{ \pm}^{n}$ sends $\partial \Delta^{n}$ to $S_{n-1}$, and we claim that the degree of $\psi_{ \pm}^{n}$ is one. Indeed, the faces of $\partial \Delta^{n}$ are given by one of the conditions: $x_{0}=0, x_{1}=0, \ldots, x_{n}=0$. Since $\psi_{ \pm}^{n}\left(0, x_{1}, \ldots, x_{n}\right)=\psi_{\mp}^{n-1}\left(x_{1}, \ldots, x_{n}\right)$ and $\psi_{ \pm}^{n}\left(x_{0}, \ldots, x_{n-1}, 0\right)=\psi_{ \pm}^{n-1}\left(x_{0}, \ldots, x_{n-1}\right)$, the map $\psi_{ \pm}^{n}$ sends the faces $x_{0}=0$ and $x_{n}=0$ to the two $n$-1-dimensional cells of $S_{n-1}$, and the other faces to the $n-2$-skeleton of $S_{n-1}$. Therefore $\operatorname{deg} \psi_{ \pm}^{n}=1$.

Since the attaching maps of two $n$-dimensional discs $\Delta^{n}$ to $S^{n-1}$ have degree one, $S_{n}$ is $n$-dimensional sphere.

One can also consider the space of piecewise constant functions on the circle with values \pm 1 and at most $n$ intervals of constant sign ( $n$ even). Such a space is also homeomorphic to $S^{n}$ : cut the circle at, say, point 0 to obtain a piecewise constant function on an interval with at most $n+1$ intervals of constant sign, and apply Theorem 4 .

\section{Proof of the converse Ghys theorem}

Let us start with a reformulation described in [8].

A diffeomorphism $f: \mathbb{R P}^{1} \rightarrow \mathbb{R P}^{1}$ has a unique lifting to a homogeneous (of degree one) area preserving diffeomorphism $F$ of the punctured plane. If $f$ is a projective transformation then $F \in \mathrm{SL}(2, \mathbb{R})$. Let $x$ be the angular parameter on $\mathbb{R P}^{1}$ so that $x$ and $x+\pi$ describe the same point. Then $(x, r)$ are the polar coordinates in the plane and

$$
F(x, r)=\left(f(x), r f^{\prime-1 / 2}(x)\right)
$$


Let $\gamma$ be the image of the unit circle under $F$; this is a centrally symmetric curve that bounds area $\pi$. The curve $\gamma$ satisfies the differential equation

$$
\gamma^{\prime \prime}(x)=-k(x) \gamma(x)
$$

where $k$ is a $\pi$-periodic function called the potential. The relation of the potential with the Schwarzian derivative is as follows:

$$
k=\frac{1}{2} S(f)+1 \text {. }
$$

In particular, the zeros of the Schwarzian corresponds to the values 1 of the function $k$ (indeed, if $k(x) \equiv 1$ then $\gamma$ is a central ellipse, $F \in \operatorname{SL}(2, \mathbb{R})$ and $f$ is a projective transformation).

Thus we arrive at the following reformulation of Theorem 2: if a function $k-1$ on $\mathbb{R P}^{1}$ changes sign at least four times then there exists an orientation preserving diffeomorphism $\varphi$ of the projective line such that the function $\bar{k}=\varphi^{*}(k)$ is the potential of a centrally symmetric closed parametric curve $\gamma$ in the punctured plane bounding area $\pi$, that is, a curve satisfying the differential equation $\gamma^{\prime \prime}(x)=$ $-\bar{k}(x) \gamma(x)$.

The proof consists of the same four steps as in Example 1.1.

Step 1. Let $k_{1}, k_{2}$ be two positive numbers satisfying $k_{1}>1, k_{1}+k_{2}=2$ and both sufficiently close to 1 . We claim that there exists a $\pi$-periodic step function $h(x)$ with four intervals of constant values $k_{1}, k_{2}, k_{1}, k_{2}$ on $[0, \pi]$ such that the corresponding solution of the differential equation (2) is a closed curve.

To prove this, consider the frame $F(x)=\left(\gamma(x), \gamma^{\prime}(x)\right)$. The differential equation (2) rewrites as

$$
F^{\prime}(x)=F(x) A(x)
$$

where

$$
A(x)=\left(\begin{array}{cc}
0 & -k(x) \\
1 & 0
\end{array}\right) .
$$

Equation (3) defines a curve on the group $\operatorname{SL}(2, \mathbb{R})$; the curve $\gamma$ is centrally symmetric and closed iff $F(\pi)=-F(0)$. Let us refer to the last equality as the monodromy condition.

Let the desired step function $h$ have intervals of constant values of lengths $t_{1}, t_{2}, t_{3}, t_{4}$ with $t_{1}+t_{2}+t_{3}+t_{4}=\pi$. For a constant potential $k$, equation (3) is easily solved:

$$
F(x)=F(0) e^{x A}=F(0)\left(\begin{array}{cc}
\cos (\sqrt{k} x) & -\sqrt{k} \sin (\sqrt{k} x) \\
\frac{1}{\sqrt{k}} \sin (\sqrt{k} x) & \cos (\sqrt{k} x)
\end{array}\right) .
$$

It follows that the monodromy condition is

$$
e^{t_{1} A} e^{t_{2} B} e^{t_{3} A} e^{t_{4} B}=-E
$$

where

and $E$ is the unit matrix.

$$
A=\left(\begin{array}{cc}
0 & -k_{1} \\
1 & 0
\end{array}\right), \quad B=\left(\begin{array}{cc}
0 & -k_{2} \\
1 & 0
\end{array}\right)
$$


Let us look for a solution satisfying $t_{3}=t_{1}, t_{4}=t_{2}$; then $t_{1}+t_{2}=\pi / 2$. Set $\alpha=t_{1} \sqrt{k_{1}}, \beta=t_{2} \sqrt{k_{2}}$. A direct computation shows that (4) is satisfied once

$$
\tan \alpha \tan \beta=\sqrt{k_{1} k_{2}} \text {. }
$$

The constraint on $\alpha$ and $\beta$ is

$$
\frac{\alpha}{\sqrt{k_{1}}}+\frac{\beta}{\sqrt{k_{2}}}=\frac{\pi}{2}
$$

If $\alpha$ is close to $\pi / 2$ then the left hand side of (5) is greater, and if $\alpha$ is close to 0 then it is smaller than the right hand side. It follows that (5) has a solution.

Step 2. Since $k(x)-1$ changes sign at least four times, there is a constant $c>0$ such that $k$ takes the values $1+c, 1-c, 1+c, 1-c$ at points, say, $x_{1}, x_{2}, x_{3}, x_{4}$. Let $k_{1}=1+c, k_{2}=1-c$, and let $h$ be the step function from Step 1. For every $\varepsilon>0$, there exists a diffeomorphism $\varphi \in \operatorname{Diff}_{+}\left(\mathbb{R P}^{1}\right)$ which stretches neighborhoods of the points $x_{1}, \ldots, x_{4}$ so that the function $\varphi^{*}(k)$ is $\varepsilon$-close in measure to $h$.

Step 3. Similarly to Step 3 in Section 2, consider a 3-parameter family of diffeomorphisms $\psi_{\alpha} \in \operatorname{Diff}\left(\mathbb{R P}^{1}\right)$ that change the intervals of constant values of the step function $h$. Given $\alpha$, consider the function $\psi_{\alpha}^{*}(h)$ as the potential of equation (3) with the initial condition $F(0)=E$. The formula $G(\alpha)=F(\pi)$ defines a smooth map $D^{3} \rightarrow \mathrm{SL}(2, \mathbb{R})$ that takes the origin to the matrix $-E$.

Lemma 4.1. The differential $d G$ is non-degenerate at the origin.

Proof. Stretch the intervals of constant values of the potential function to $t_{i}+$ $\varepsilon s_{i}, i=1,2,3,4$; the vector $s=\left(s_{1}, s_{2}, s_{3}, s_{4}\right), s_{1}+s_{2}+s_{3}+s_{4}=0$, is interpreted as a tangent vector to $D^{3}$ at the origin. Using the formula for monodromy (4), we compute

$$
-d G(s)=s_{1} A+s_{4} B+s_{2} e^{t_{1} A} B e^{-t_{1} A}+s_{3} e^{t_{2} B} A e^{t_{2} B}
$$

where $A, B, t_{1}, t_{2}$ are as in Step 1 . We need to check that the linear map $d G: \mathbb{R}^{4} \rightarrow$ $\mathrm{sl}_{2}$, given by (6), is surjective and that its kernel is transverse to the hyperplane $s_{1}+s_{2}+s_{3}+s_{4}=0$. Both claims follow, by a direct computation, from the explicit formulas for the matrices $A, B$ and their exponents given in Step 1.

Step 4. This last step is identical to Step 4 in Section 2: replace the potential $h$ in the definition of the map $G$ in Step 3 by $\varphi^{*}(k)$. We obtain a new monodromy map $\bar{G}: D^{3} \rightarrow \mathrm{SL}(2, \mathbb{R})$ whose image contains the matrix $-E$. The corresponding curve closes up, and we are done.

Remark 4.2. The Ghys theorem is closely related to the four vertex theorem in the hyperbolic plane [11]. Let $\gamma$ be an oval in $H^{2}$. Each tangent line to $\gamma$ intersects the circle at infinity in two points, and this defines a circle diffeomorphism $f_{\gamma}$. In the projective model of hyperbolic geometry, the circle at infinity is represented by a conic in $\mathbb{R P}^{2}$. A conic has a canonical projective structure, hence $f_{\gamma}$ can be viewed as a diffeomorphism of $\mathbb{R P}^{1}$. Singer's theorem asserts that the zeros of 
the Schwarzian $S\left(f_{\gamma}\right)$ correspond to the vertices of $\gamma$ (in the hyperbolic metric, of course); see [7] for a discussion.

Note, however, that a converse four vertex theorem for the hyperbolic plane does not hold in the same way as in the Euclidean plane: if the positive curvature function is too small then the relevant curve in the hyperbolic plane does not close up.

\section{Problems and conjectures}

There are many other results extending the four vertex theorem. In each case, it is interesting to find the converse theorem; we mention but a few.

Problem 1. Another classical theorem of Mukhopadhyaya is that a plane oval has at least six affine vertices (also known as sextactic points). An affine vertex is a point at which the curve is abnormally well approximated by a conic: at a generic point, a conic passes through five infinitesimally close points of the curve, whereas at an affine point, this number equals six. Every oval $\gamma$ can be given an affine parameterization such that $\operatorname{det}\left(\gamma^{\prime}(x), \gamma^{\prime \prime}(x)\right)$ is constant. Then $\gamma^{\prime \prime \prime}(x)=-k(x) \gamma^{\prime}(x)$ where the function $k(x)$ is called the affine curvature. The affine vertices are the critical points of the affine curvature; see, e.g., [7].

A conjectural converse theorem asserts that if a periodic function $k$ has at least six extrema then there exists a plane oval $\gamma$ whose affine curvature at point $\gamma(x)$ is $k(x)$ (of course, here $x$ is not necessarily an affine parameter).

Problem 2. The four vertex theorem has numerous discrete versions; see, e.g., [7, 9] for surveys and references. For example, let $P$ be a convex $n$-gon with vertices $x_{1}, \ldots, x_{n}$. Assume that $n \geq 4$ and that no four consecutive vertices lie on a circle. Consider the circles circumscribing triples of consecutive vertices $x_{i-1} x_{i} x_{i+1}$, and assume that the center of this circle lies inside the cone of vertex $x_{i}$ (such a polygon is called coherent). Let $r_{1}, \ldots, r_{n}$ be the cyclic sequence of the radii of the circles. Then the sequence $r_{1}, \ldots, r_{n}$ has at least two local maxima and two local minima.

A conjectural converse theorem asserts that if a cyclic sequence $r_{1}, \ldots, r_{n}$ has at least two local maxima and two local minima then it corresponds, as described above, to a coherent convex polygon.

Another version of discrete four vertex theorem concerns the circles tangent to the triples of consecutive sides of a polygon: the radii of such inscribed circles also form a cyclic sequence with at least two local maxima and two local minima. One conjectures that a converse theorem holds as well.

\section{Acknowledgments}

It is a pleasure to acknowledge interesting discussions with D. Fuchs, H. Gluck, D. Khavinson, V. Ovsienko, I. Pak, A. Pinkus, R. Schwartz, D. Singer and V. Totik. The author was partially supported by an NSF grant DMS-0555803. 


\section{References}

[1] B. Dahlberg, The converse of the four vertex theorem. Proc. Amer. Math. Soc. 133 (2005), 2131-2135.

[2] D. DeTurck, H. Gluck, D. Pomerleano and D. S. Vick, The four vertex theorem and its converse. Notices Amer. Math. Soc. 54 (2007), 192-207.

[3] H. Gluck, The converse to the four vertex theorem. Enseign. Math. 17 (1971), 295309.

[4] C. Hobby and J. Rice, A moment problem in $L_{1}$ approximation. Proc. Amer. Math. Soc. 16 (1965), 91-106.

[5] J. Matoušek, Using the Borsuk-Ulam Theorem. Lectures on Topological Methods in Combinatorics and Geometry. Springer, Berlin, 2003.

[6] M. Krein and A. Nudelman, The Markov Moment Problem and Extremal Problems. Amer. Math. Soc., Providence, RI, 1977.

[7] V. Ovsienko and S. Tabachnikov, Projective Differential Geometry, Old and New: From Schwarzian Derivative to Cohomology of Diffeomorphism Groups. Cambridge Univ. Press, 2005.

[8] V. Ovsienko and S. Tabachnikov, Sturm theory, Ghys theorem on zeroes of the Schwarzian derivative and flattening of Legendrian curves. Selecta Math. 2 (1996), 297-307.

[9] I. Pak, Introduction to Discrete and Polyhedral Geometry. A book to appear.

[10] A. Pinkus, A simple proof of the Hobby-Rice theorem. Proc. Amer. Math. Soc. 60 (1976), 82-84.

[11] D. Singer, Diffeomorphisms of the circle and hyperbolic curvature, Conform. Geom. Dyn. 5 (2001), 1-5.

[12] V. Totik, A tale of two integrals. Amer. Math. Monthly 106 (1999), 227-240.

Serge Tabachnikov

Department of Mathematics

Pennsylvania State University

University Park, PA 16802, USA

e-mail: tabachni@math.psu.edu

To access this journal online: www.birkhauser.ch/jfpta 